\title{
Is Hypertrophic Osteoarthropathy Associated with Smoking?
}

\author{
Sahin $\mathrm{A}^{1 *}$, Pepeler $\mathrm{MS}^{2}$, Ergene $\mathrm{E}^{2}$, Kucuksahin $\mathrm{O}^{3}$ and Sahin $\mathrm{M}^{4}$
}

${ }^{1}$ Rheumatology Division, Sanliurfa Education and Research Hospital, Sanliurfa, Turkey

${ }^{2}$ Internal Medicine Unit, Sanliurfa Education and Research Hospital, Sanliurfa, Turkey

${ }^{3}$ Rheumatology Department, Ankara University Medical Faculty, Ankara, Turkey

${ }^{4}$ Physical Therapy and Medicine Unit, Sanliurfa Education and Research Hospital, Sanliurfa, Turkey

\begin{abstract}
Hypertrophic Osteoarthropathy (HOA) is a disease of the joints and bones characterized by advanced clubbing that is bulbous enlargement of the ends of one or more fingers or toes; proliferation and oedema of connective tissue resulting loss of the normal angle between the skin and nail, excessive sponginess of the nail base of the digits of the hands and feet. There is painful periosteal proliferation of long bones such as the radius, ulna, or tibia in clubbing, and the periostitis is often associated with mild fever and arthralgia or joint effusion. There are two accepted types of HOA, primary and secondary. The former is usually inherited and hereditary (idiopathic), while the secondary type seen as a concurrent manifestation of another diseases. Herein, we present a 23-year-old man diagnosed with primary HOA and had only smoking history. After examining the patient, we have concluded that there is possibility of relationship between the HOA and smoking history.
\end{abstract}

Keywords: Hypertrophic osteoarthropathy; Primary; Smoking

\section{Introduction}

Hypertrophic Osteoarthropathy (HOA) was firstly defined as paraneoplastic syndrome by Marie and Bambarger in the late $19^{\text {th }}$ century. HOA can occur primary (idiopathic) or secondary. In the primary type of $\mathrm{HOA}$ is also known as pachydermoperiostosis or Toraine-Solante-Gole Syndrome (5\%) predominantly affecting men, with a hereditary aspect and there is no underlying factor or disease $[1,2]$. Beside that, HOA may take place secondary to another disease, usually pulmonary. Due to that in the past, it was also known as hypertrophic pulmonary osteoarthropathy. Of the total cases, $90 \%$ is associated with malignancies, and more rarely it is sometimes related with pulmonary pathologies including cystic fibrosis, pulmonary abscess, bronchiectasis, emphysema, cyanotic congenital heart disease [3].

Although the HOA is seen about $17 \%$ of lung cancer cases but the prognostic significance remains very poor $[4,5]$. We herein present a 23-year-old man diagnosed with primary HOA and has only smoking history.

\section{Case Report}

A 23 year-old man admitted to our outpatient service with a three years history of gradually increasing pain and swelling in fingertips. There was not a family history of clubbing; familial or congenital clubbing can have its onset in the late teens or later. The patient's history and careful physical examination revealed ongoing pain and tenderness complaints in wrist and ankle but there was no pruritus, eruption and oral ulcers. Unlike other cases, patients questioning indicated the absence of back pain, morning stiffness, whitening of fingers in cold weather, heel pain, bruising, blushing, nail pitting, and tenderness in other joints, night sweating, fever, weight loss. Inflammatory bowel diseases history, tuberculosis, and brucellosis were not identified, except smoking history.

Pulmonary pathologies including cystic fibrosis, pulmonary abscess, bronchiectasis, emphysema, cyanotic congenital heart disease were observed in several cases but it was not detected in this patient. There was no history of rheumatic diseases, chronic inflammatory diseases or infection (etc. tuberculosis) in family members. Although there was no obvious "leonine facies" appearance the same as seen as in leprosy in physical examination, there was a valuable decaying fat pad in his face. Clubbing and hour-glass nails in both hand fingers, and there was hypertrophy-pachyderma (Figure 1). Laboratory markers including, complete blood count, urine analyses, erythrocyte sedimentation rate, $\mathrm{C}$ - reactive protein, and ferritin levels were normal. Ferritin plays a role in humoral theory of HOA. Calcium, phosphorus, alkaline phosphatase, and other liver and kidney functions were in normal ranges. Thyroid functions and Parathyroid Hormone (PTH) values $(57.45 \mathrm{pg} / \mathrm{ml}, \mathrm{N}$ : 15-65) were also in normal ranges.

Rheumatoid Factor (RF), Anti Nuclear Antibody (ANA), other auto-antibodies including anti-scl-70, anti-centromere, anti-SS-A, anti-SS-B and other Extractable Nuclear Antigen (ENA) antibodies, anti-cyclic citrulinized peptide (anti-CCP) and anti-ds-DNA were negative. C3, C4, and IgA, IgG, IgM concentrations were in parallel with the reference ranges. These tests were done for differential diagnosis of (peripheral) arthritis. Screening tests such as HBsAg and anti-HCV (for
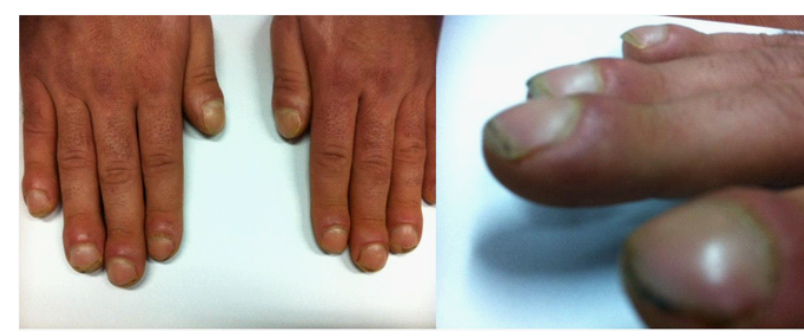

Figure 1: Beside hour-glass nails, loss of angle and thickening the nail bed, and there was clubbing, hypertrophy-pachyderma and acropachy observed.

*Corresponding author: Ali Sahin, Rheumatology Division, Sanliurfa Education and Research Hospital, Sanliurfa/Turkey, 63100, Fax: +90 41431868 12; E-mail: dralsahin@hotmail.com

Received April 05, 2012; Accepted May 05, 2012; Published May 18, 2012

Citation: Sahin A, Pepeler MS, Ergene E, Kucuksahin O, Sahin M (2012) Is Hypertrophic Osteoarthropathy Associated with Smoking? J Clin Case Rep 2:145 doi:10.4172/2165-7920.1000145

Copyright: ( 2012 Sahin A, et al. This is an open-access article distributed under the terms of the Creative Commons Attribution License, which permits unrestricted use, distribution, and reproduction in any medium, provided the original author and source are credited. 
rulling out these as aetiologies of liver cirrhosis), anti-HIV and VDRL other orphan diseases like brucellosis and familial mediterranean fever test results were negative but PPD test results was $20 \mathrm{~mm}$ that above the reference threshold of $5 \mathrm{~mm}$. Examination of the peripheral blood smear as an important indicator of hematological diseases revealed clustered and enough platelet, and there were no abnormal giant platelets. Due to absence of symptoms in patient's history and clinical findings, we have not intended to investigate acromegaly. However, we know that growth hormone also play a role in humoral theory of HOA.

Evaluation of pulmonary X-ray graphy and High-Resolution Computed Thorax Tomography (HRCT)-abdominal tomography findings were normal. There were periarticular osteopenia, evident swelling in distal phalanges' soft tissues, periosteal reaction, and thickening of the periosteum in tubular bones (Figure 2). Evidently there were no cardio-vascular pathologic finding in vascular examination and his echocardiography was normal. Presence of expected symptoms including clubbing in fingers, male gender, and absence of other diseases with similar clinical and laboratory findings, the patient diagnosed with primary hypertrophic osteoarthropathy based on solely smoking history beside those leading symptoms. Colchicine has anti-inflammatory, and immunomodulatory effects. So, we started colchicine and non-steroidal anti-inflammatory drug and added to follow-up list. His pain and other symptoms were alleviated by colchicine and indomethacine.

\section{Discussion}

Hypertrophic Osteoarthropathy (HOA) or "acropachy" is characterized by extreme proliferation of skin and bone at the distal parts of extremities with skin changes. Excessive proliferation in distal parts of fingers known as "clubbing" and in the further stages of the diseases radiological changes including periostosis of tubular bones, and synovial effusions observed [6]. Acropachy is triggered through a mechanism of multi-factorial mediators where von Willebrand antigens, megakaryocyte fragmentation in pulmonary vascular vein, Vascular Endothelial Growth Factor (VEGF) released by interaction and activation of endothelial cells with giant platelets in systemic circulation play vital roles in the pathogenesis of HOA [7].

The VEGF released from platelet that is induced with hypoxia is a potent angiogenic and stimulates osteoblastic differentiation. In primary and secondary HOA, there is increased serum VEGF levels and accumulation of VEGF in stroma. There is prevalent perspective

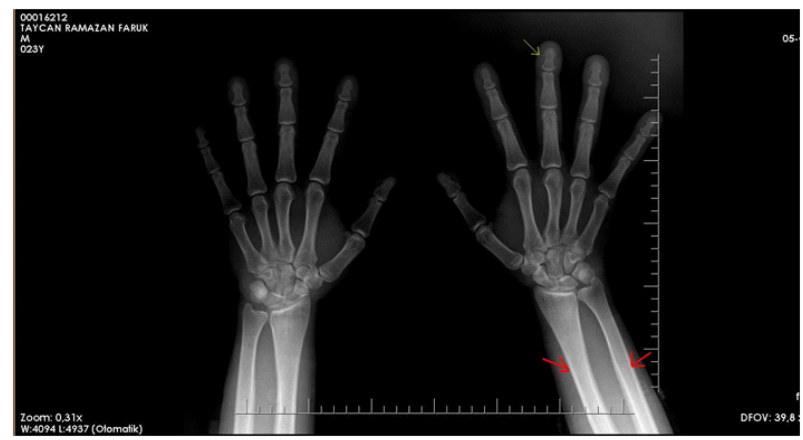

Figure 2: X-Ray demonstrates periarticular osteopenia, evident swelling in distal phalanges' soft tissues (yellow arrow), periosteal reaction, and periosteal proliferation of the tubular bones (red arrows). that VEGF plays a key role in the development of the acropachy might be seen in infective endocarditis, Grave's disease and mesothelioma. Neurogenic and humoral theories have been responsible from the development of osteoarthropathy. Markedly improvement of symptoms with vagotomy and atropine confirms neurogenic theory $[8,9]$.

Certain substances including prostaglandin, ferritin, bradykinin, estrogen, and growth hormone could play important role in humoral theory. VEGF inhibition with octreotide could be valuable improvement in HOA findings [10-12]. Disappearance of angle in the perimeter of the nail bed at the distal interphalangeal joints and swelling is generally evident. HOA is generally seen in muscleless joints of hand and foot extremities. In the early onset period of diseases, long bones like tibia, fibula femur and ulna involved. In the short period firstly metaphysis and distal diaphysis on the bones are affected, and epiphysis affected in the long period. There would be palpation sensitive massive joint effusions in these regions, but synovial hypertrophy is unusual. There were no inflammatory synovitis, and effusion developed as a reaction to periostosis.

Pachydermoperiostosis or primary HOA is characterized with diffuse skin hypertrophy, pachyderma, cutis verticis gyrate, seborrhea or acne. There is not a specific laboratory marker unique to HOA but there would be abnormal laboratory findings of concomitant diseases. Although the toes affected, typical joint space protected, erosion and periarticular osteopenia is not observed. Periosteal involvement can be observed with radionuclide bone scans. Without irregular periosteal proliferation in the beginning of the epiphyseal region in the distal of phalanges and tibular bones and without severe narrowing of medullary cavity, clubbing, thickening and rarely acro-osteolysis observed in these patients $[13,14]$.

HOA can be seen also such diseases that pulmonary fibrosis, liver cirrhosis, inflammatory bowel diseases, chronic infections, thalassemia, achalasia, thymoma, esophageal cancer, and other mediastinal cancers, presence of $\mathrm{A}-\mathrm{V}$ fistula, polyposis, excessive use of laxatives and POEMS (polyneuropathy, organomegaly, endocrinopathy, M-protein, skin changes) syndrome. Treatment regimen generally determined in accordance with the other concomitant manifestations. Primary goal of the treatment need to be solving cardiac problems, removing malign tumors and endocarditis. Beside analgesic and non-steroidal anti-inflammatory drugs (NSAIDs), colchicine, pamidronate, bisphosphonate (drugs that inhibit osteoclastic resorption, rarely leads to VEGF inhibition), subcutaneous octreotide, and bevacizumab could be postulated [15-18].

Clinical and laboratory evidence presented with this case are very close to leading pathogenesis of primary HOA. Despite to a $20 \mathrm{~mm}$ induration of tuberculin skin test, there was not a tuberculosis history of patient and his family. Because of normal lung X-Ray and HRCT scan further investigations (quantiferon etc.) concerning the TB was not held. In the further stages of follow-up patient's VEGF levels couldn't be detected and there was only smoking history related to HOA that could be used as primary diagnosis.

Here are some questions the whole discussion raises "Can smoking unilaterally be enough to trigger HOA?" or "Smoking affects genetic liability" or "Smoking has effects on epigenetic changes that are heritable". As we know, some rheumatic diseases eg. rheumatoid arthritis is associated with smoking. Smoking is an aetiological factor 
Citation: Sahin A, Pepeler MS, Ergene E, Kucuksahin O, Sahin M (2012) Is Hypertrophic Osteoarthropathy Associated with Smoking? J Clin Case Rep 2:145. doi:10.4172/2165-7920.1000145

developing the disease. Consequently, specific unique smoking history without any co-morbidity could be enough to be an aetiological factor for primary HOA in this case or not? In fact, HOA which is a clinical/ radiological diagnosis, further studies with larger cohorts need to hold to verify findings presented in this case and relation between smoking and developing HOA.

\section{References}

1. von Bamberger E (1889) Veranderungen der rohrrenknochen bei bronchiektasie. Wien Klin Wochenschr 2: 226

2. Marie P, De I (1890) Osteo-arthropathie hypertrophiante pneumique. Rev Med Paris 10: 1-36

3. Vogl A, Goldfischer S (1962) Pachydermoperiostosis: Primary or idiopathic hypertrophic osteoarthropathy. Am J Med 33: 166-187.

4. Martinez-Lavin M (1992) Cardiogenic hypertrophic osteoarthropathy. Clin Exp Rheumatol 10: 19-21.

5. Yang WC, Lin SC, Liu TC, Chen CJ, Yen JH, et al. (2003) Clubbed fingers and hypertrophic soteoarthropathy in a patient with squamous cell carcinoma of the lung. Kaohsiung J Med Sci 19: 183-187.

6. Martinez-Lavin M, Matucci-Cerinic M, Jajic I, Pineda C (1993) Hypertrophic osteoarthropathy: consensus on its definition, classification, assessment and diagnostic criteria. J Rheumatol 20: 1386-1387.

7. Olan F, Portela M, Navarro C, Gaxiola M, Silveira LH, et al. (2004) Circulating vascular endothelial growth factor concentrations in a case of pulmonary hypertrophic osteoarthropathy. Correlation with disease activity. J Rheumatol 31: 614-616.

8. Kurzrock R, Cohen PR (2002) Cutaneous paraneoplastic syndromes associated with bronchogenic carcinoma. Clin Chest Med 23: 257-264.

9. Nashitz JE, Rosner I, Rozenbaum M, Elias N, Yeshurun D (1995) Cancer- associated rheumatic disorders: clues to occult neoplasia. Semin Arthritis Rheum 24: 231-241.

10. Wegrowski Y, Gillery P, Serpier H, Georges N, Combemale P, et al. (1996) Alteration of matrix macromolecule synthesis by fibroblasts from a patient with pachydermoperiostosis. J Invest Dermatol 106: 70-74.

11. Matucci-Cerinic M, Martinez-Lavin M, Rojo F, Fonseca C, Kahaleh BM (1992) von Willebrand factor antigen in hypertrophic osteoarthropathy. J Rheumato 19: $765-767$.

12. Silveira LH, Martinez-Lavin M, Pineda C, Fonseca MC, Navarro C, et al. (2000) Vascular endothelial growth factor and hypertrophic osteoarthropathy. Clin Exp Rheumatol 18: 57-62.

13. Martinez-Lavin M, Pineda C, Valdez T, Cajigas C, Weisman M, et al. (1988) Primary hypertrophic osteoarthropathy. Semin Arthritis Rheum 17: 156-162.

14. Pineda C, Fonseca C, Martinez-Lavin M (1990) The spectrum of soft tissue and skeletal abnormalities in hypertrophic osteoarthropathy. J Rheumatol 17: $626-632$.

15. Amital H, Applbaum YH, Vasiliev L, Rubinow A (2004) Hypertrophic pulmonary osteoarthropathy: control of pain and symptoms with pamidronate. Clin Rheumatol 23: 330-332.

16. Blackwell N, Bangham L, Hughes M, Melzack D, Trotman I (1993) Treatment of resistant pain in hypertrophic pulmonary arthropathy with ketorolac. Thorax 48: 401.

17. Kozak KR, Milne GL, Morrow JD, Cuiffo BP (2006) Hypertrophic osteoarthropathy pathogenesis: a case highlighting the potential role for cyclooxygenase-2-derived prostaglandin E2. Nat Clin Pract Rheumatol 2: 452-456.

18. Ooi A, Saad RA, Moorjani N, Amer KM (2007) Effective symptomatic relief of hypertrophic pulmonary osteoarthropathy by video-assisted thoracic surgery truncal vagotomy. Ann Thorac Surg 83: 684-685. 